\title{
Degradação Ultra-Sônica da Borracha Natural de Seringueira (Hevea Brasiliensis) em Solução Toluênica: Estudo por GPC
}

\author{
Antonio S. G. Magalhães e Judith P. de A. Feitosa
}

Resumo: A redução da massa molar e consequentemente da viscosidade são efeitos observados quando soluções poliméricas são submetidas a ultrasom de alta intensidade. A degradação por ultrasom de soluções toluênicas de borracha natural da seringueira (Hevea brasiliensis) foi estudada por GPC. Os efeitos da temperatura e do tempo de irradiação sobre a polidispersão da borracha foram estudados.

Palavras-chave: Ultra-som, cavitação, degradação, borracha natural, polidispersão

\section{Introdução}

O efeito mais evidente da exposição de uma solução polimérica à irradiação ultra-sônica de alta energia é a diminuição de sua viscosidade e a redução de massa molar ${ }^{[1,2]}$. O processo foi primeiro descrito nos anos trinta. Atualmente constata-se um evidente crescimento pelo interesse no assunto, motivado pela maior disponibilidade e menor custo de equipamentos de ultra-som, principalmente os de grande escala.

Não há evidências de que existe interação entre a onda de som e a cadeia polimérica. De acordo com o mecanismo mais difundido na literatura, a redução da massa molar ocorre como um resultado da interação das ondas de ultra-som com o solvente. Muitos dos efeitos físicos e químicos causados pelo ultra-som são atribuídos ao processo de cavitação (crescimento e rápido colap- so de microbolhas) ocasionados quando as ondas se propagam através da solução. Isto gera em frações de segundos temperaturas e pressões elevadíssimas. No caso de polímeros, é o alto gradiente de cisalhamento formado em torno das microbolhas colapsadas, o maior responsável pelo processo de degradação, bem como as ondas de choque formadas. O mecanismo geralmente aceito é que os segmentos das cadeias poliméricas próximas ao ponto de colapso se movem com velocidade superior àquelas dos segmentos mais distantes. A tensão produzida por esta diferença gera um stress e a posterior quebra da cadeia, ocasionando a redução da massa molar ${ }^{[2,3]}$. Sendo assim, muitas das propriedades resultantes do processo podem ser explicadas em termos do efeito de cavitação.

A degradação ultra-sônica é dependente de uma série de fatores como: gás dissolvido, 
solvente, temperatura, freqüência, intensidade, massa molar do polímero e concentração da solução $^{[4]}$. A relação de dependência, no entanto, ainda não está bem definida para muitos destes fatores.

Quanto ao processo de cisão da molécula existe alguma controvérsia. O processo de degradação pode ser aleatório ou não. Price e Smith ${ }^{[3]}$ consideram o processo como não aleatório com a quebra ocorrendo preferencialmente nas proximidades do meio da cadeia. Alugiev e Shibaeva ${ }^{[5]}$ verificaram a presença de inúmeros fragmentos de baixa massa molar, o que é incompatível com um processo não aleatório.

Como uma técnica para controlar massa molar, o ultra-som oferece a possibilidade de produzir materiais funcionalizados ${ }^{[6]}$. O ultra-som tem sido usado com alguma freqüência como ferramenta para a obtenção de amostras de polímeros com massa molar menor do que a de um polímero mãe. É evidente que neste processo não deverá ocorrer mudanças estruturais no decorrer da degradação $^{[7]}$. Em alguns trabalhos foram citadas alterações estruturais, por exemplo na degradação de borracha butílica em tetracloreto de carbono (CC14) e em p-xileno ${ }^{[5]}$.

Desde a década de trinta que a borracha natural vem sendo matéria de pesquisa científica. A partir de 1944 houve um avanço significativo, quando Armstrong et $\mathrm{al}^{[8]}$ propuseram que na vulcanização, o enxofre era o responsável pela formação de ligações cruzadas entre as moléculas poliméricas da borracha.

Apesar da grande variedade de materiais sintéticos, a borracha natural ainda tem hoje um importante papel tanto no âmbito acadêmico, quanto tecnológico. A borracha natural foi conhecida, durante muito tempo, como um poli-1,4-cisisopreno puro. Tanaka ${ }^{[9]}$ verificou, através de 13C-NMR, que a estrutura do hidrocarboneto da borracha natural tem variações que, apesar de pequenas, são extremamente importantes para definir o comportamento do polímero e suas propriedades.

No presente trabalho objetiva-se estudar o processo de degradação ultra-sônica da borracha natural (BN) de seringueira - Hevea brasiliensis - em solução, a partir de dados obtidos por cromatografia de permeação em gel - GPC.

\section{Experimental}

A borracha natural (BN) de seringueira (Hevea brasiliensis), tipo CCB - 1 (Crepe Clara Brasileira) já purificada, foi fornecida pela Usina Planalto Paulista. A BN foi sempre mantida sob refrigeração $\left(\sim-10^{\circ} \mathrm{C}\right)$, na ausência de luz e em atmosfera de nitrogênio. Foram empregados clorofómio e tolueno da REAGEN e tolueno para cromatografia da VETEC.

As irradiações foram efetuadas em banho de limpeza ultra-sônico (freqüência nominal $40 \mathrm{kHz}$ e potência $50 \mathrm{~W}$ ), modelo THORNTON T14, contendo água destilada como meio para a propagação das ondas sonoras. A temperatura do banho ultra-sônico foi controlada através de uma serpentina de vidro e um banho ultratermostatizado modelo 111 da FANEM.

Em cada experimento foram utilizados $25 \mathrm{~mL}$ de uma solução de $\mathrm{BN}(0,4 \mathrm{~g} / \mathrm{dL}$, saturada com N2) em um erlenmeyer de $50 \mathrm{~mL}$, o qual foi posto sempre na mesma posição, centralizado, no banho ultra-sônico.

As irradiações foram desenvolvidas nos intervalos de tempo de $30,45,60,75,90,120,240$, 360,480 e 600 minutos nas temperaturas de 15 , 35,45 e $55^{\circ} \mathrm{C} \pm 1{ }^{\circ} \mathrm{C}$. Em todos os experimentos o sistema foi mantido aberto.

Utilizou-se um sistema cromatográfico tipo HPLC da SHIMADZU, consistindo de uma bomba modelo LC-10AD operando isocraticamente, e um detetor de índice de refração modelo RID-6A, ambos da SHIMADZU. Uma coluna da Waters, tipo Styragel HMW 6E com 6,8 X 300 mm, empacotada com copolímero estireno-divinilbenzeno (SDVB). A fase móvel utilizada foi tolueno, a um fluxo de $1 \mathrm{~mL} / \mathrm{min}$ sob temperatura ambiente $\left(\sim 25^{\circ} \mathrm{C}\right)$. Foram empregados padrões de poliestireno (Shodex - Showa denko), Mw variando de $1,13 \times 104$ a 3,15 x $106 \mathrm{~g} / \mathrm{mol}$.

\section{Resultados e discussão}

Diante da possibilidade de estar havendo degradação térmica na $\mathrm{BN}$ durante o processo de degradação mecânica (ultra-som), a solução de BN foi submetida a aquecimento de $55^{\circ} \mathrm{C}$, mantendo as mesmas condições de degradação por ultra-som (solvente, concentração da $\mathrm{BN}$, volume de solu- 


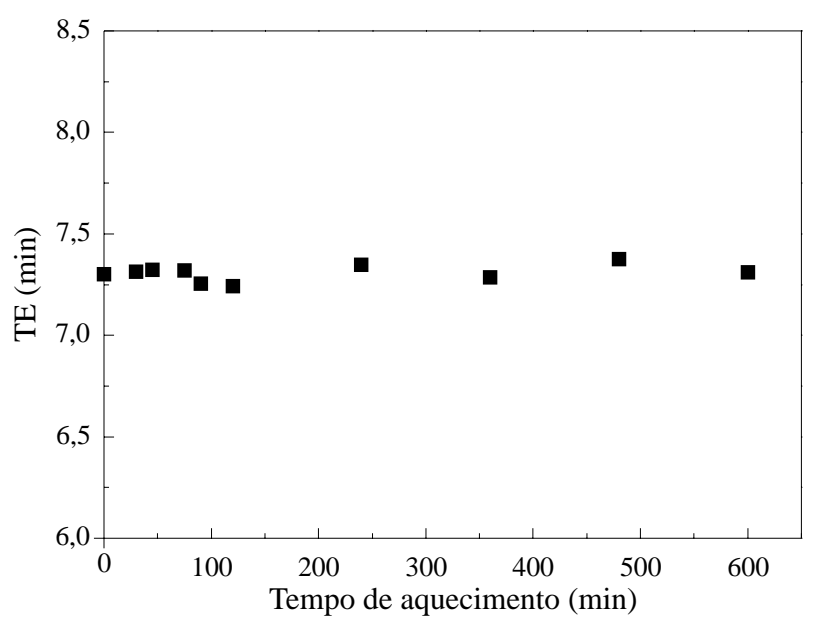

Figura 1. Variação dos tempos de eluição contra tempo de reação para a solução de $\mathrm{BN}$ aquecida à $55^{\circ} \mathrm{C}$.

ção, tempo de aquecimento). O gráfico da Figura 1 , mostra a tendência na variação dos tempos de eluição com o tempo de aquecimento.

Praticamente não há variação, e os valores oscilam em torno do valor médio 7,308 minutos. A comparação com o valor inicial (7,302 min) e a não constatação de tendência de aumento no tempo de eluição indicam que não houve degradação térmica nas condições estudadas, ou seja, a contribuição da degradação térmica é insignificante no processo de degradação ultra-sônica da $\mathrm{BN}$.

A partir de uma curva de calibração construída com padrões de poliestireno e dos tempos de eluição das amostras de BN sonicadas, foi possível determinar os valores das massas molares de pico (Mpk) da BN para os respectivos tempos e temperaturas de sonicação.

Analisando a tendência seguida pelas massas molares durante o processo de sonicação, tem-se uma idéia da cinética de degradação (Figura 2). As massas molares de pico (Mpk) diminuem rapidamente durante os primeiros estágios da degradação. Posteriormente, este decréscimo fica menos acelerado, tendendo a um valor limite. É evidente a dependência do processo de degradação com a temperatura. A velocidade de degradação é bem menor na sonicação à $55^{\circ} \mathrm{C}$, do que na sonicação à $15^{\circ} \mathrm{C}$. Este efeito é bem mais definido para maiores tempos de irradiação. Observa-se que, ao final de 120 minutos, há uma sensível diferença de $1,4 \times 10^{5} \mathrm{~g} / \mathrm{mol}$ entre as massas molares da BN irradiada à 55 e $15^{\circ} \mathrm{C}$. Este fato se deve à maior mobilidade das cadeias e à diminuição na intensidade de cavitação com a elevação da temperatu-

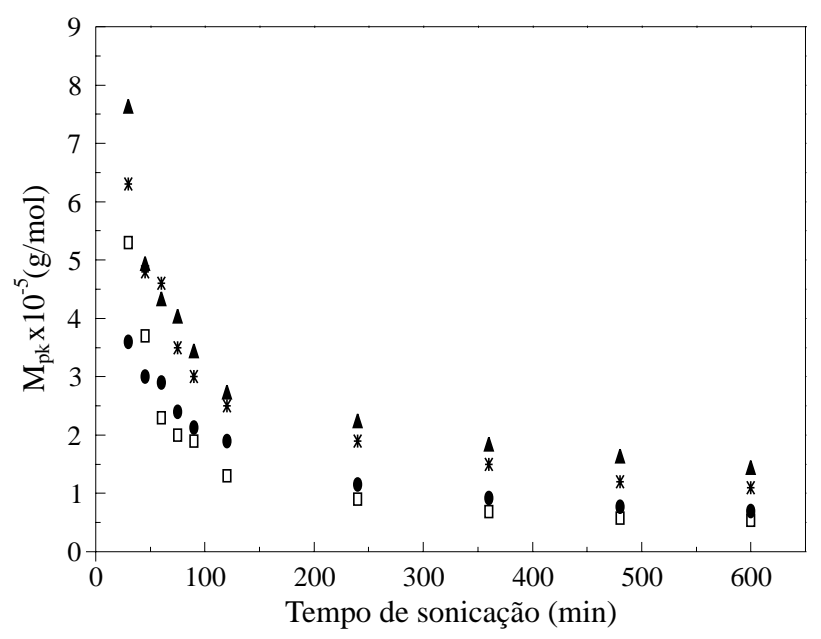

Figura 2. Variação dos valores de Mpk com a temperatura e tempo de sonicação. ( $\square$ ) $15^{\circ} \mathrm{C}$; (•) $35^{\circ} \mathrm{C}$; (g) $45^{\circ} \mathrm{C}$; (s) $55^{\circ} \mathrm{C}$.

ra, bem como à diminuição da intensidade das ondas de choque e do gradiente de cisalhamento $^{[10-12]}$. A intensidade da cavitação é função da pressão de vapor do solvente, variando em ordem inversa. Com a elevação da temperatura, ocorrerá uma aumento na pressão de vapor do solvente, que acarretará uma maior quantidade de vapor nas bolhas de cavitação, que produzirá uma redução na pressão e temperatura máximas atingidas no momento da implosão e amortecerá o processo de implosão, diminuindo a velocidade das moléculas do solvente e a intensidade das ondas de choque.

Apesar de terem sido observados deslocamentos consideráveis nos picos dos cromatogramas à medida que se aumentava o tempo de irradiação ultra-sônica, é importante salientar que uma melhor eficiência seria obtida com a utilização de colunas acopladas em série. Pelo fato de ter sido utilizada apenas uma coluna, mesmo com larga faixa de massa molar, não foi possível determinar valores confiáveis de $\mathrm{Mw}$ e $\mathrm{Mn}$ para a $\mathrm{BN}$ degradada. Curvas de distribuição de massa molar foram construídas a partir das massas e as respectivas alturas, obtidas diretamente do software (CLASS LC10). As áreas foram normalizadas, possíbilitando análises qualitativas.

$\mathrm{O}$ efeito da irradiação ultra-sônica às temperaturas de $15,35,45$ e $55{ }^{\circ} \mathrm{C}$, na distribuição de massa molar, pode ser analisado, respectivamente, a partir das Figuras 3 a 6.

Observa-se, nas Figuras 3 a 6, que as curvas de distribuição se deslocam para regiões de massas molares menores à medida que se aumenta $o$ 


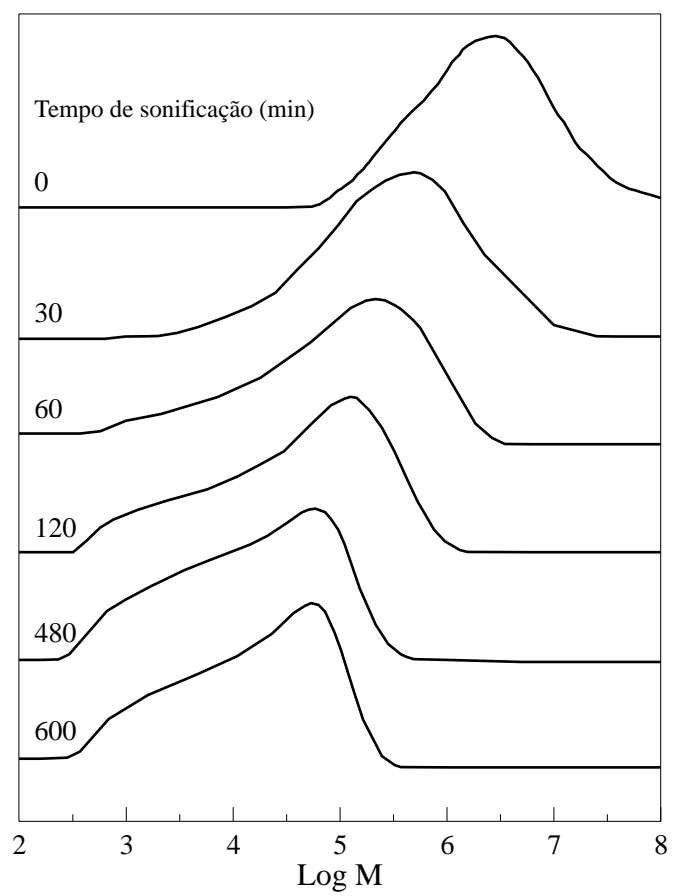

Figura 3. Curvas normalizadas de distribuição de massa molar para $\mathrm{BN}$ sonicada à $15^{\circ} \mathrm{C}$ em vários intervalos de tempo

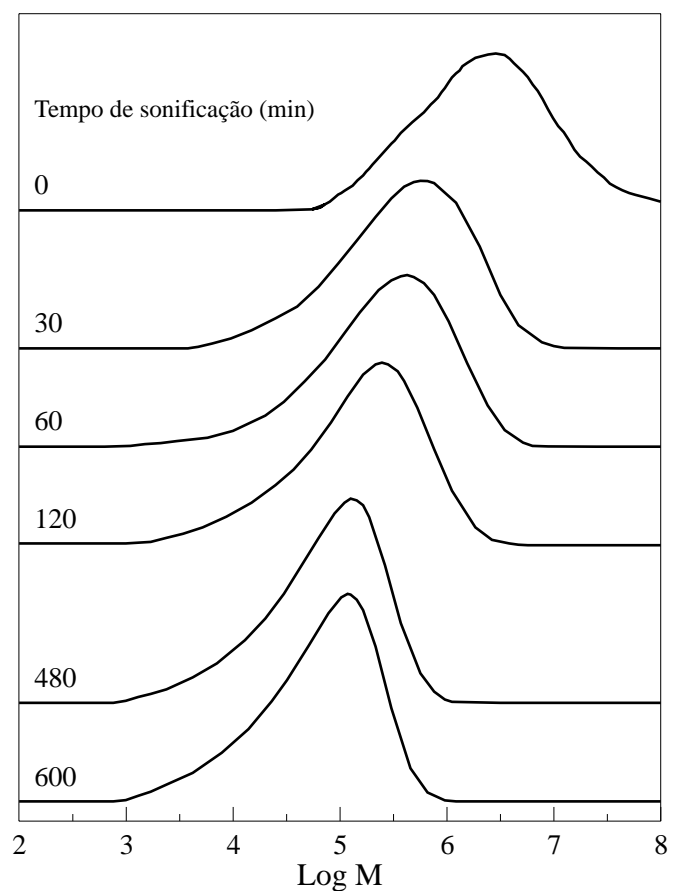

Figura 5. Curvas normalizadas de distribuição de massa molar para $\mathrm{BN}$ sonicada à $45^{\circ} \mathrm{C}$ em vários intervalos de tempo.

tempo de irradiação. Nas quatro temperaturas de sonicação, verifica-se que a diferença no deslocamento das curvas para os tempos de irradiação de 480 e 600 minutos é muito pequena, indicando que o processo atingiu o limite de degradação.

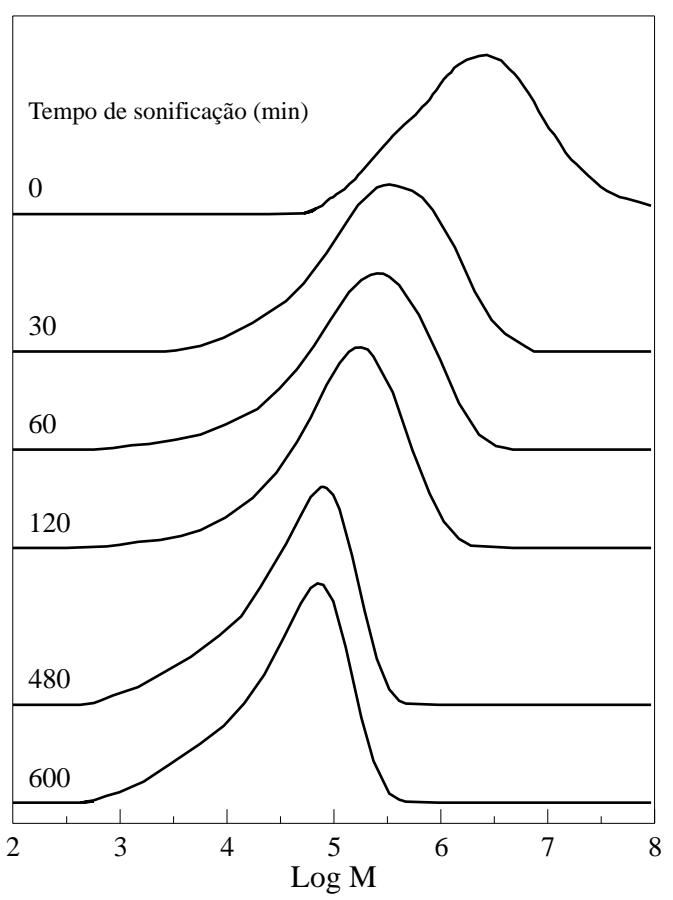

Figura 4. Curvas normalizadas de distribuição de massa molar para $\mathrm{BN}$ sonicada à $35^{\circ} \mathrm{C}$ em vários intervalos de tempo.

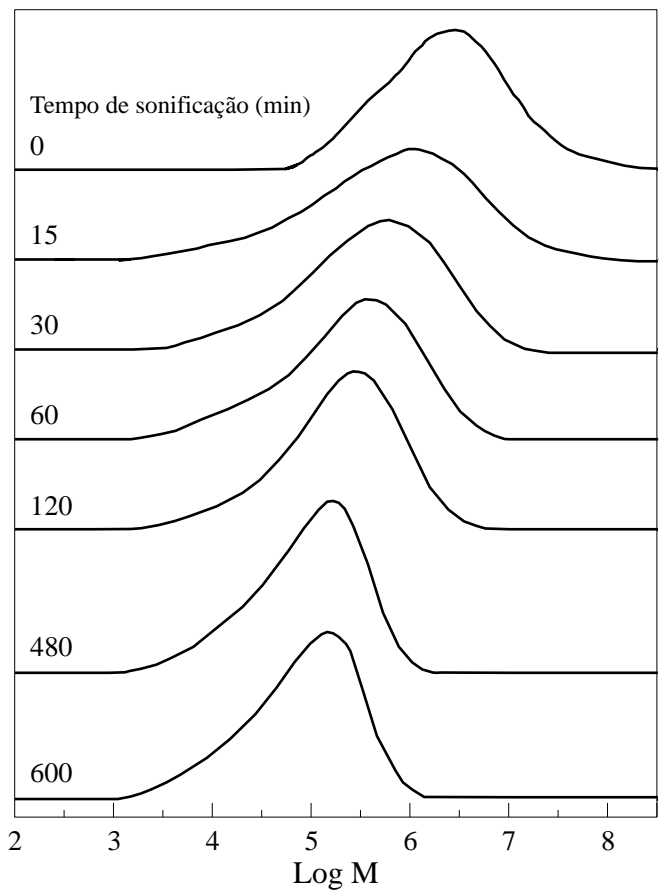

Figura 6. Curvas normalizadas de distribuição de massa molar para $\mathrm{BN}$ sonicada à $55^{\circ} \mathrm{C}$ em vários intervalos de tempo.

Diferentemente das outras temperaturas, à $15^{\circ} \mathrm{C}$ observa-se uma certa diferença no perfil das curvas de distribuição de massa molar. A partir de 60 minutos começa a surgir um leve ombro em torno de $\mathrm{M}=10^{3} \mathrm{~g} / \mathrm{mol}(\log \mathrm{M}=3)$, que é intensi- 
ficado para tempos mais longos de irradiação, aumentando a proporção de material com massa molar entre $10^{3}$ e $3,2 \times 10^{3} \mathrm{~g} / \mathrm{mol}$. Este fato pode ser indicativo de uma distribuição bimodal, indício de uma cisão não aleatória.

A polidispersão ou o grau de distribuição de massa molar pode ser qualitativamente indicado através da largura de meia altura do cromatograma de GPC ${ }^{[13]}$. Fukumore et al. ${ }^{[14]}$ utilizaram este artifício para determinar a polidispersão de ácidos polidesoxiribonucleicos. Com o mesmo objetivo, foram determinados os valores de largura de meia altura $\left(\mathrm{L}_{1 / 2}\right)$ das curvas de distribuição mostradas nas Figuras 3 a 6. Na Figura 7 pode-se observar a variação dos valores de $\mathrm{L}_{1 / 2}$ com o tempo de sonicação e a temperatura.

Os valores das larguras de meia banda são um indicativo de que a polidispersão diminuiu com o tempo de sonicação, tanto à 35 como à $45^{\circ} \mathrm{C}$. À 55 ${ }^{\circ} \mathrm{C}$ verifica-se uma ligeira elevação inicial no valor de $\mathrm{L}_{1 / 2}$ seguido de uma diminuição, voltando a tendência normal de decréscimo. Para $15^{\circ} \mathrm{C}$, ocorre exatamente o oposto, ou seja, há uma elevação na polidispersão com o tempo de sonicação. $\mathrm{O}$ aparecimento do ombro em regiões de menor massa molar nas curvas de distribuição de massa, referente à temperatura de $15{ }^{\circ} \mathrm{C}$, contribuiu para o aumento na polidispersão. A Figura 8 apresenta de forma mais detalhada a tendência inicial na variação da polidispersão para a $\mathrm{BN}$ sonicada à $55^{\circ} \mathrm{C}$, acrescentando-se o ponto correspondente a 15 minutos de sonicação. Fica, então, mais evidente o aumento da polidispersão nos primeiros estágios de degradação.

A elevação inicial da polidispersão pode estar

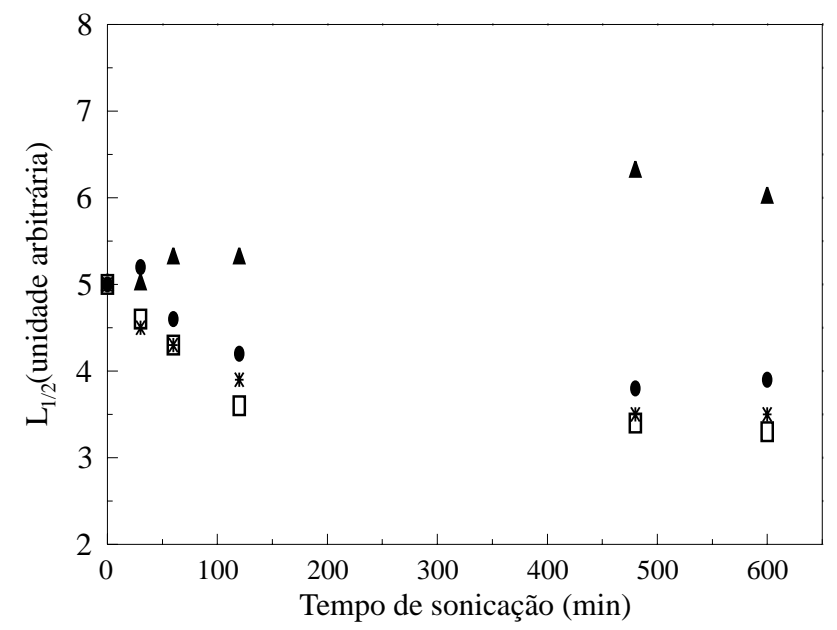

Figura 7. Efeito do tempo de sonicação sobre a largura de meia altura de GPC $\left(\mathrm{L}_{1 / 2}\right)$ da BN. (s) $15{ }^{\circ} \mathrm{C}$; ( $\left.\square\right) 35{ }^{\circ} \mathrm{C}$; (g) $45{ }^{\circ} \mathrm{C}$; (1) $55^{\circ} \mathrm{C}$

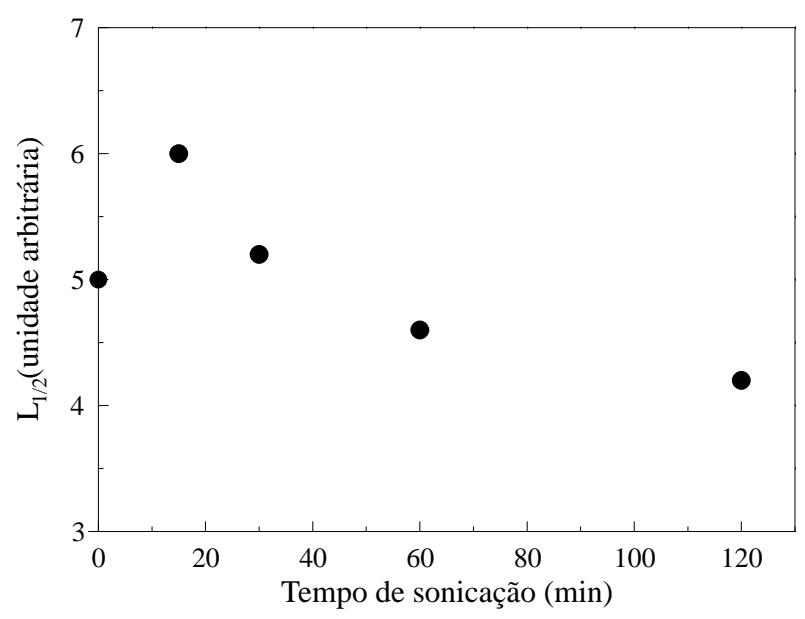

Figura 8. Efeito do tempo de sonicação inicial sobre a largura de meia banda de GPC $\left(\mathrm{L}_{1 / 2}\right)$ da BN sonicada à $55^{\circ} \mathrm{C}$.

relacionada com possíveis recombinações de macroradicais, formados no início do processo, entre si e com moléculas ainda não afetadas pela degradação mecânica, produzindo material com maior massa molar, possivelmente mais ramificados.

Este aumento foi observado para outros sistemas. Koda et al. ${ }^{[16]}$ utilizando um transdutor PZT côncavo operando em $500 \mathrm{kHz}$ e $80 \mathrm{~W}$, verificaram inicialmente uma elevação nos valores de $\mathrm{Mw} /$ Mn e posterior decréscimo, quando submeteram xantana a irradiação ultra-sônica em solução.

\section{Conclusão}

A contribuição da energia térmica na degradação da borracha natural é insignificante, sendo o ultra-som o maior responsável pela diminuição da massa molar do polímero.

A polidispersão diminuiu com o tempo de sonicação nas temperaturas de 35 e $45^{\circ} \mathrm{C}$. à $15^{\circ} \mathrm{C}$ houve um aumento.

$\mathrm{O}$ processo de degradação à $15^{\circ} \mathrm{C}$ parece ter um comportamento bimodal, característico de cisão não aleatória, com quebra preferencialmente no ponto médio da cadeia.

A $55^{\circ} \mathrm{C}$ foi observado uma elevação da polidispersão nos primeiros 30 minutos de sonicação, ocasionado, possivelmente, por recombinações de macroradicais.

\section{Agradecimentos}

CAPES, CNPq e FUNCAP 


\section{Referências Biblográficas}

1. Basedow, A. M., Ebert, K. H., Adv. Polym. Sci., 2283 (1977).

2. Price, G. J., Adv. Sonochem. ,1 231 (1990).

3. Price, G. J., Smith, P. F., Eur. Polym. J. , 29 (1993) 419.4. Lorimer, J. P., Mason, T. J., ed., em Sonochemistry: The uses of ultrasound in Chemistry, Royal Society of Chemistry, Cambridge, cap. 9 (1990).

5. Aliguliev, R. M., Shibaeva, A. A., Int. Polym. Sci. and Technol., 19 T/97 (1992).

6. Price, G. J., Smith, P. F., Polymer, 344111 (1993).

7. Ueda, K., Ochial, H., Itaya, T., Yamaoka, K., Polymer, 33429 (1992).
8. Armstrong, R. T., Litlle, J. R., Doak, K. W., Rubb. Chem. Technol., 17788 (1944).

9. Tanaka, Y., Proc. Int. Rubb. Conf., Kuala Lumpur, 273 (1985).

10. Carlsson, D. J., Wiles, D. M., Macromol. Chem., c14 65 (1976).

11. Binder, J. L., J. Polym. Sci., 137 (1963).

12. Salomon, G., Schee, A. C., J. Polym. Sci., 14 (1953).

13. Bac, N. V. B., Terlemezyan, L., Mihailov, M., J. Appli. Plym. Sci.,50 845 (1993).

14. Fukudome, K., Kumamoto, Y., Yamaoka, K., Polym. J., 27 (1995) 101.

15. Koda, S., Mori, H., Matsumoto, K., Nomura, H., Polymer, 3531 (1994). 